\title{
Optimal load scheduling within a microgrid including reliability aspects
}

\author{
Zubin Japa Balan, Prabodh Bajpai* \\ Electrical Engineering Department, Indian Institute of Technology, Kharagpur, PIN:721302, India
}

\begin{abstract}
Establishment and running of microgrids involve considerable investment. In order to justify the investment, the economic benefits arising out of microgrid have to be studied. In this work, an analysis of profit to the loads is performed, including reliability parameters like probability of interruption and average duration of interruption. A case study is presented, for a microgrid operating along with the main grid having a time of day tariff for power supply. When there is interruption of main grid supply, the microgrid generators meet the load. The loads are scheduled such that the probability weighted average electricity cost is the minimum. Then the profit for the loads because of the microgrid is calculated. It is inferred from the simulation results that, the microgrid is beneficial to the loads, subject to reliability conditions, tariff structure and worth of energy for the loads.
\end{abstract}

Keywords: Benefit, heuristic algorithms, load scheduling, microgrid, reliability

\section{Introduction}

Microgrids are increasingly being considered as options to increase the reliability of power supply, as it can operate either in synchronism with the main grid or in stand-alone mode [1]. The economic benefits have to be greater than the investment made, for the system to be viable [2]. Since most of the loads have a time of day tariff, the loads have to be scheduled, such that their electricity cost is the minimum [3]. Reliability parameters could be used for load scheduling and eventually to calculate the benefit to the loads [4], [5].

A microgrid has been considered in the present work, which has a time of day tariff when power is drawn from main grid. There are several generators within this microgrid, which could support the load, during the period of interruption of power supply from the main grid [6]. The loads have to be scheduled; such that their probability weighted average electricity cost is the minimum. Moreover, the interruption can occur at any instant of time. So, the problem has been formulated to calculate the probability weighted average electricity cost, and use this to evaluate the profit to the loads due to microgrid. The load scheduling problems is non linear and hence heuristic algorithms like genetic algorithm (GA) [7] and particle swarm optimization (PSO) [8] have been used to solve this problem. The result obtained with heuristic algorithms is compared with dynamic programming (DP) algorithm [9] results.

\section{Problem Formulation}

There will be several loads in any microgrids, which need not be supplied for all twenty four hours of a day. Such loads can be scheduled to specific time slots such that the total electricity charge paid by the microgrid loads is the minimum. Load scheduling is done so as to take advantage of the time of day tariff structure [10]. The probability of interruption of power supply from main grid, during which microgrid operates in stand-alone mode, also need to be considered for load scheduling. The suitability of a 
particular load schedule is defined using 'probability weighted average electricity cost' representing the average electricity charge incurred by microgrid loads after considering probability of interruption.

Probability weighted electricity cost, with interruption at a particular hour, is the product of electricity cost incurred by the microgrid loads and the probability of interruption at that hour. Probability weighted electricity cost is different when interruption happens at different hours. So it is calculated for all possible hours of interruption. Then their average gives the probability weighted average electricity cost.

The research problem formulated in this work has two parts. First the microgrid loads are scheduled based on probability weighted average electricity cost and second, the additional profit to the loads, due to the microgrid, is calculated. The loads are scheduled so that the probability weighted average electricity cost is the minimum. Once the loads are scheduled in the optimal manner, then the actual profit to the loads is calculated as the difference between 'worth of energy' for the loads and the electricity charges paid by them. 'Worth of energy' of a load is the net commercial gain of the loads in hourly basis excluding the electricity charges.

If the interruption of supply from main grid occurs once in a day with the probability $\lambda$, then $1-\lambda$ is the probability that interruption will not occur in the day, and the probability weighted average electricity cost can be expressed

$$
C=(1-\lambda) \cdot N C I+\lambda \cdot C I
$$

where $C I=$ Electricity cost for a day when interruption occur in that day, and $N C I=$ Electricity cost, if no interruption occur in a day.

Let there be $p$ tariff periods in a day. For each of the tariff periods represented by variable $x$, the interruption can start in any hour ( $y=1$ to $y=T(x)$ ), where $T(x)$ is the duration of $x$ th tariff period So the second part of Eq. (1) can be modified to express probability weighted average electricity as

$$
C=(1-\lambda) \cdot(N C I)+\lambda / 24 \cdot \sum_{x=1}^{p} \sum_{y=1}^{T(x)}\left(C I_{x, y}\right)
$$

where $C I_{x, y}=$ electricity cost when interruption starts at $y$ hours after the beginning of $x$ th tariff period. Here it is assumed that the probability for interruption to occur at each of the 24 hours of the day is same and is equal to $\lambda / 24$. $N C I$ and $C I$ of Eq. (2) can be evaluated as shown below

Let the load schedule be represented by load decision matrix $\mathbf{S}$. The rows of $\mathbf{S}$ matrix represent hours and the columns represent status of the loads. If a load is scheduled to operate in an hour, then the corresponding element of the $\mathbf{S}$ matrix is 1 . If the load is not scheduled in that hour, the corresponding element value is 0 . Let there be $L$ non-continuous loads within the microgrid, which are indexed using $i$ and the demand of $i$ th load be $P_{i}$ in $\mathrm{kW}$. The total demand of non-interruptible loads is taken as $P_{c}$ in $\mathrm{kW}$ and the average duration of interruption of the upstream network is $I_{u p}$ in hours. Let $F_{e}(k)$ be the energy charge in $k$ th tariff period in INR/kWh, and $F_{d}(k)$ be the demand charge in $k$ th tariff period in INR/kW. Hours in a day is indexed by $a$. Let the tariff periods be represented by $k$ ( $k=1$ means 1 st tariff period, $k$ $=2$ means 2nd tariff period and so on up to $k=p$ ), and $T(k)$ be the duration of $k$ th tariff period.

If there is no interruption during a day, then the electricity cost for the loads, $N C I$ is the sum of energy and demand charges and is given by

$$
N C I=\sum_{k=1}^{p}\left(\sum_{a=1}^{24}\left\{P_{c}+\sum_{i=1}^{L}\left[S(a, i) P_{i}\right]\right\}\right) F_{e}(k)+\sum_{k=1}^{p}\left(P_{c}+\max \left\{\sum_{i=1}^{L}\left[S(a, i) P_{i}\right]\right\}\right) F_{d}(k)
$$

Now, if there is interruption at 1st hour during 1st tariff period of the day, then electricity cost can be denoted as $C I_{1,1}$ in Eq. (2) and it can be expressed as the sum of $C_{1}, C_{2}$ and $C_{3}$, where $C_{1}$ is the electricity cost during the period of interruption of the main grid, $C_{2}$ is the electricity cost after the period of interruption till the end of the tariff period during which the interruption ends and $C_{3}$ is the electricity cost for the remaining tariff periods after the end of the interruption. $C_{1}, C_{2}$ and $C_{3}$ are calculated as shown below 


$$
C_{1}=\sum_{k=m g}\left(\sum_{a=1}^{I_{u p}}\left\{P_{c}+\sum_{i=1}^{L}\left[S(a, i) P_{i}\right]\right\}\right) F_{e}(k)+\sum_{k=m g}\left(P_{c}+\max \left\{\sum_{i=1}^{L}\left[S(a, i) P_{i}\right]\right\}\right) F_{d}(k)
$$

Assuming the interruption ends during the $r$ th tariff period, then

$$
C_{2}=\sum_{k=r}\left(\sum_{a=I_{u p}+1}^{T(1)+T(2) \ldots T(k)}\left\{P_{c}+\sum_{i=1}^{L}\left[\left(S(a, i) P_{i}\right]\right\}\right) F_{e}(k)+\sum_{k=r}\left(P_{c}+\max \left\{\sum_{i=1}^{L}\left[S(a, i) P_{i}\right]\right\}\right) F_{d}(k)\right.
$$

Assuming the remaining tariff periods after the end of the interruption is $k^{\prime}$, then

$$
\begin{aligned}
& C_{3}=\sum_{k=k^{\prime}}^{p}\left(\sum_{a=T(1)+T(2) \ldots T(k-1)+1}^{24}\left\{P_{c}+\sum_{i=1}^{L}\left[S(a, i) P_{i}\right]\right\}\right) F_{e}(k)+\sum_{k=k^{\prime}}^{p}\left(P_{c}+\max \left\{\sum_{i=1}^{L}\left[S(a, i) P_{i}\right]\right\}\right) F_{d}(k) \\
& C I_{1,1}=C_{1}+C_{2}+C_{3}
\end{aligned}
$$

$C I_{1,1}$ gives the electricity cost, when interruption begins in the 1st hour of the day. The probability of this condition is $\lambda / 24$. Similarly electricity cost when interruption happens at each hour, has to be calculated and multiplied with their respective probabilities to get the probability weighted average electricity cost.

If the interruption begins $y$ hours after the beginning of $x$ th tariff period, and ends $h$ hours after the beginning of $r$ th tariff period, then $r$ and $h$ can be calculated using the flowchart shown in Fig. 1. Within the flowchart, $n$ counts the tariff periods after the beginning of interruption while variable $f$ counts the hours after the beginning of interruption. The hours are incremented till it becomes greater than the duration of interruption $\left(I_{u p}\right)$ and the corresponding tariff period is recorded as $r$. Variable $g$ is a reverse hour counter, which is initialised at the hour at which interruption ends. Then the hours already included in previous tariff periods are subtracted to get the hours of interruption in the $r$ th tariff period only. This is recorded as $h$.

The problem is formulated to minimise probability weighted average electricity cost (Eq. (2)) to get the load decision matrix $\mathbf{S}$ representing optimal load schedule.

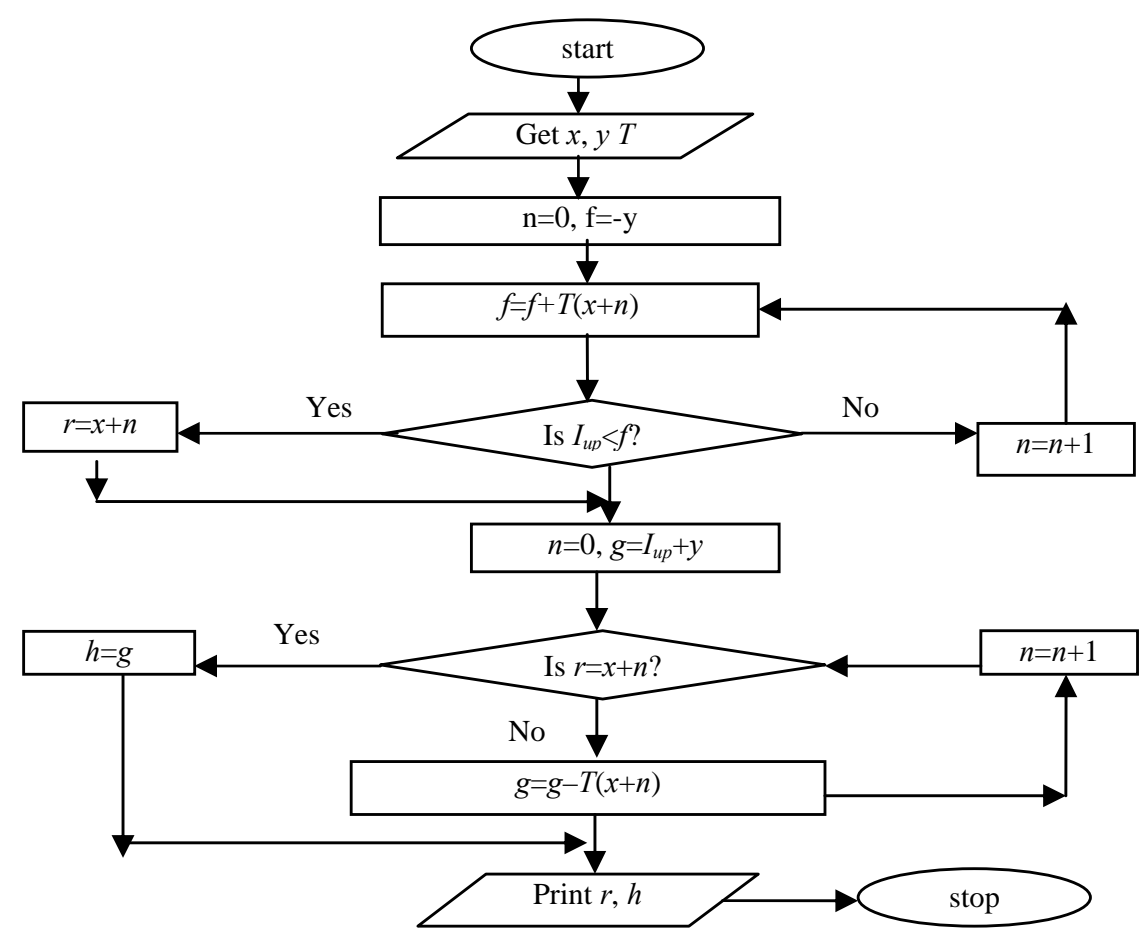

Fig. 1. Flowchart for calculation of $r$ and $h$ 
The daily profit of the utility on receiving continuous power supply can be calculated by subtracting electricity cost from worth of energy $(W)$. The worth of energy is the net commercial gain by the load on hourly basis after excluding the electricity charges.

The utility's profit on having a microgrid can be expressed as

$$
\text { Uprofit }_{M G}=24 \mathrm{~W}-\mathrm{C}
$$

If there is no microgrid, then the utility's profit is given by

$$
\text { Uprofit }_{N M G}=[W-(E / 24)]\left[24-\left(\lambda I_{u p}\right)\right]
$$

where $E$ is average daily electricity cost based on main grid tariff. The net profit for the loads due to microgrid can be calculated as follows

$$
\text { Uprofit }_{\text {net }}=\text { Uprofit }_{M G}-\text { Uprofit }_{N M G}
$$

Eq. (10) is used for the calculation of net profit for the loads, due to microgrid operation during main grid interruption after optimal load scheduling.

\section{Solution Methodology}

Initially the loads have to be scheduled, for calculating the probability weighted average electricity cost and net profit for the loads. This is performed by finding the value of load decision matrix $\mathbf{S}$ such that the probability weighted average electricity cost of the loads (Eq. (2)) is the minimum. Since the problem formulation is non liner, heuristic algorithms PSO and GA are used to solve the problem. The result obtained with heuristic methods is compared with DP algorithm results.

PSO has been defined as a heuristic algorithm where trial solutions are considered as particles distributed in the search space. At each point, the particles have fitness values, depending on the suitability of the position. Each particle would remember its best position and the best position among all particles [8], [11].

GA belong to the larger class of evolutionary algorithms, which generate solutions to optimization problems using techniques inspired by natural evolution, such as mutation, selection, and crossover [12]. The trial solutions are called individuals and the individual components of the solution are called chromosomes. The best individuals would be selected into a gene pool (selection). Parts of them could be exchanged randomly (cross over) or new chromosomes could be added randomly (mutation).

In dynamic programming (DP) algorithm all the possible solutions would be explored. The unfeasible solutions are removed by applying constraints at each step. The costs calculated at each step have to be arranged in tables. Then the path with minimum cost could be selected.

Table 1. Fixed interval loads

\begin{tabular}{ccc}
\hline \hline Load Index & Load $(\mathrm{kW})$ & Interval (hours) \\
\hline 1 & 40 & $1-8$ \\
2 & 40 & $20-24$ \\
\hline \hline
\end{tabular}

Table 2. Short duration loads

\begin{tabular}{ccc}
\hline \hline Load Index & Load (kW) & Duration Required (hours) \\
\hline 3 & 260 & 12 \\
4 & 100 & 8 \\
5 & 150 & 4 \\
\hline \hline
\end{tabular}

Table 3. Tariff Structure

\begin{tabular}{cccc}
\hline \hline Tariff period (hours) & Duration (hours) & Demand charge (INR/kW) & Energy charge(INR/kWh) \\
\hline 1 to 4 & 4 & 100 & 5 \\
5 to 16 & 12 & 150 & 7.5 \\
17 to 24 & 8 & 200 & 10 \\
Microgrid stand-alone & - & 250 & 12.5 \\
\hline \hline
\end{tabular}




\section{Case Study}

A case study has been formulated, with a microgrid consisting of interruptible and non interruptible commercial loads. The non interruptible loads have to be supplied for all the 24 hours of a day. The interruptible loads are of two kinds- either fixed intervals loads (Table 1), or any short duration loads in a day (Table 2). A time of day tariff structure has been considered, with separate charges for maximum demand, in addition to energy charges (Table 3). The probability of interruption in the upstream network has been taken as 0.2 and the average duration of interruption as 8 hours. It has been assumed that the loads make a profit of INR $1 \times 10^{5}$ per hour after excluding the electricity charges. The objective is to schedule the loads, such that the probability weighted average electricity cost is the minimum, and calculate the net profit for the loads due to the microgrid.

\section{Simulation Result and Analysis}

The tariff is highest during the peak tariff period. Therefore if the electricity cost is to be the least, then both the maximum demand and the energy consumed during this period should be as low as possible. Thus it can be seen from Table 4 that only load 2 is scheduled during the peak tariff period (17-24 hours).

The load scheduling has been performed using GA, PSO and DP algorithms, in MATLAB. A comparison of the results is illustrated in Fig. 2 and Table 5. From Fig. 2 it is seen that the convergence characteristics is similar for both GA and PSO, but from Table 5, it can be observed that, best result for load scheduling, in terms of time of execution and memory utilisation is obtained using GA. So, GA has been used for further analysis of the result.

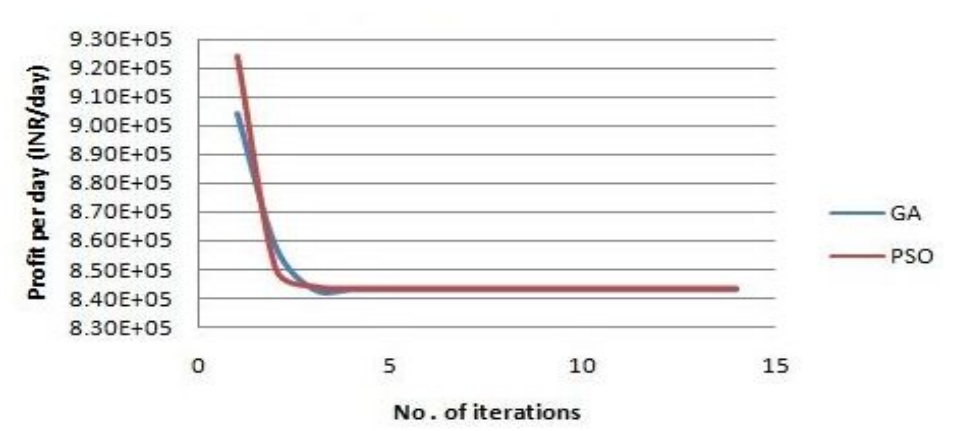

Table 4. Optimum distribution of load

\begin{tabular}{lccccc}
\hline \hline Load Index & 1 & 2 & 3 & 4 & 5 \\
\hline Hours 1-4 & 1 & 0 & 0 & 0 & 0 \\
Hours 5-8 & 1 & 0 & 1 & 1 & 0 \\
Hours 9-12 & 0 & 0 & 1 & 0 & 1 \\
Hours 13-16 & 0 & 0 & 1 & 1 & 0 \\
Hours 17-20 & 0 & 0 & 0 & 0 & 0 \\
Hours 21-24 & 0 & 1 & 0 & 0 & 0 \\
\hline \hline
\end{tabular}

Fig. 2. Convergence characteristic of GA and PSO

Table 5. Comparison of methods for load scheduling

\begin{tabular}{cccc}
\hline \hline Characteristic & Genetic Algorithm & Particle Swarm Optimisation & Dynamic Programming \\
\hline Convergence & 13 iterations & 14 iterations & - \\
Time of Execution & 3 seconds & 14 seconds & 4 hours and 32 minutes \\
Cost Calculated (INR) & 843270 & 843270 & 843270 \\
Net Profit (INR) & 17600 & 17600 & 17600 \\
Sample solutions & 100 & 1000 & All viable solutions \\
\hline \hline
\end{tabular}

\subsection{Probability of interruption of main grid and benefit for the loads}

With the increase in probability of interruption, the share of power drew from microgrid generators increases. Since the microgrid tariff is usually higher than the tariff from the main grid, the electricity charges for the loads increase with the probability of interruption of main grid and consequently, profit decreases (Fig. 3 (a)). However, if microgrid is not present, the loads would not receive any power supply during the period of interruption, and there would not be any profit for the loads during this period. Hence if microgrid is present, there is a net profit, compared to not having the power supply, and this net profit increases as the probability of interruption of power supply from the main grid increases. 


\subsection{Duration of interruption of main grid and benefit for the loads}

When the average duration of interruption increases, the power drawn from microgrid generators increases and consequently the profit to the loads decreases. However there is a net profit to the loads in having a microgrid compared to not having a microgrid, because if microgrid is present, then the loads can continue to realise their worth of energy during the period of interruption. This can be seen from Fig. 3 (b). So microgrid is preferred in areas where the average duration of interruption is high.

\subsection{Microgrid tariff and benefit for the loads}

Fig. 4 (a) shows the plot between the profit of the utility and microgrid tariff taken as the percentage of the tariff considered in the case study (Demand charge of Rs 250/kW and energy charge of Rs $6.25 / \mathrm{kWh}$ ). Since the microgrid tariff is higher than the main grid tariff, the loads will have to pay a higher electricity cost, during the periods of interruption. Higher microgrid tariff results in higher average electricity cost to the loads, which offsets the gain to the loads drive from microgrid supply during the interruption of main grid. Thus there is a trade off between microgrid tariff and net profit to the loads.

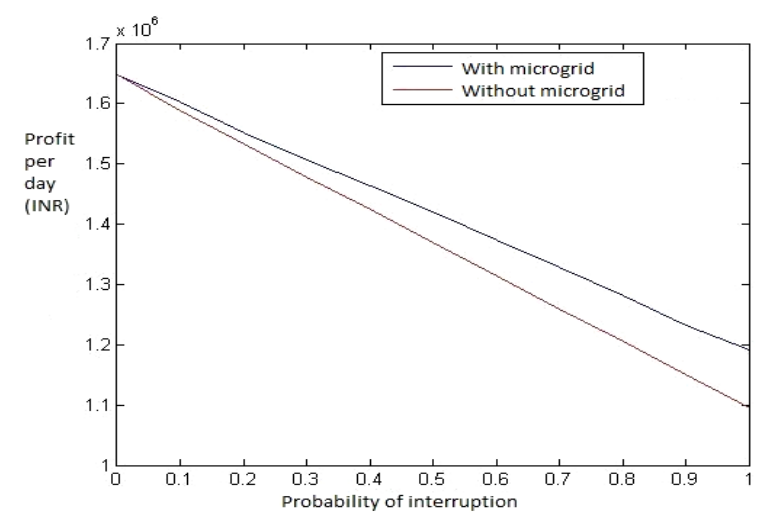

(a)

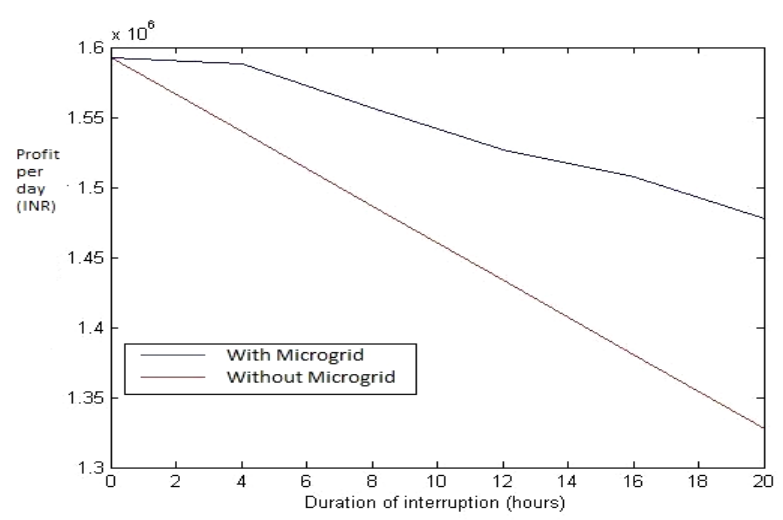

(b)

Fig. 3. Load benefit as a function of (a) Probability of interruption of main grid (b) Average duration of interruption of main grid

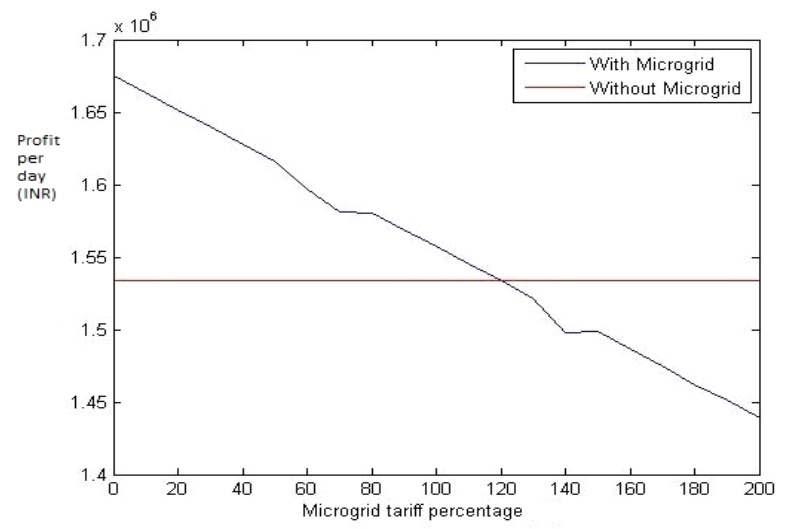

(a)

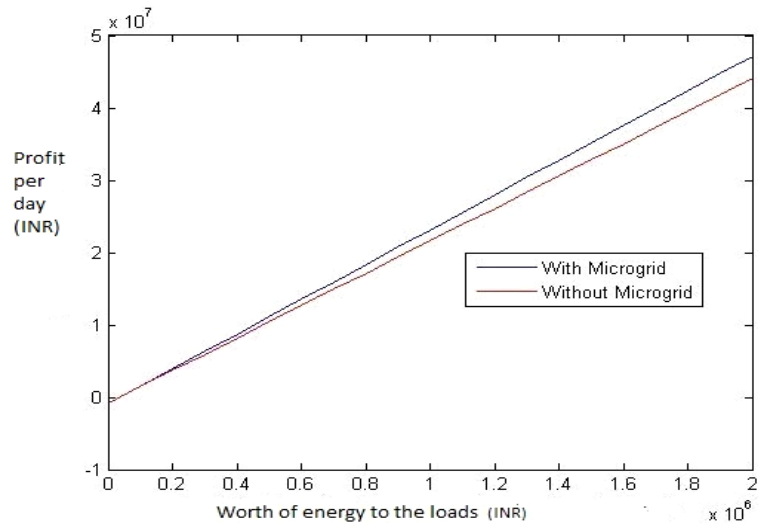

(b)

Fig. 4. Load benefit as a function of (a) Microgrid tariff (b) Worth of energy to the load

\subsection{Worth of Energy to the loads and benefit for the loads}

'Worth of energy' to a load is defined as the net commercial gain the load would derive in one hour, if power supply is available, excluding electricity cost. The net profit in having a microgrid is higher for loads with higher 'worth of energy', as they derive higher profit during the period of interruption of main grid power supply. This can be seen in Fig. 4 (b). So microgrid is more beneficial in areas having loads with higher 'worth of energy'. 


\section{Conclusion}

In this work, the mathematical formulation for evaluation of economic benefit arising out of a microgrid, in terms of profit to the microgrid loads is proposed. Profit to the loads is calculated including tariff structure of loads within the microgrid and the reliability parameters like probability of interruption and average duration of interruption of the main grid. It has been verified with a simulation case study that the microgrid provides cost advantage to loads, with the increase in reliability. The extent of economic benefit depends upon the level of interruption in the locality, microgrid tariff and the 'worth of energy' for the loads.

\section{Acknowledgements}

The authors wish to acknowledge the continuous support for this research work provided by the Indian Institute of Technology, Kharagpur, India.

\section{References}

[1] Hatziargyriou N, Asano H, Iravani R, Marnay C. Microgrids. IEEE Power an Energy Magazine, 2005; 5(4):78-94.

[2] Asanol H. Economic Analysis of microgrids. In: Proc. of Power Conversion Conference - Nagoya, 2007:654-658

[3] Ashok S, Banerjee R. Load management application for industrial sector. Applied Energy, 2000; 66(2):105-111.

[4] Costa PM. Economic analysis of microgrids including reliability aspects. In: Proc. of International Conference on Probabilistic Methods Applied to Power Systems, 2006:1-8.

[5] Costa PM, Matos MA. Reliability of distribution networks with microgrids. In: Proc. of IEEE Conference on Power Tech, 2005:1-7.

[6] Govardhan MD, Roy R. Artificial bee colony based optimum management of microgrid. In: Proc. of 11th International Conference on Environment and Electrical Engineering, 2012:334-339.

[7] Frenzel JF. Genetic algorithms, A new breed of optimisation. Potentials, IEEE, 1993; 12(3):21-24.

[8] Kennedy J. Particle swarm optimisation. In: Proc. of IEEE International Conference on Neural Networks, 1995:1942-1948.

[9] Synder Jr W, Powell DH. Dynamic programming approach to unit commitment. IEEE transaction on Power Systems,1987; 2(2):339-348.

[10] Balasubramanya C. Analysis of the indian situation for time of day tariff. In: Proc. of Seventh International Conference on Metering Apparatus and tariff for Electricity Supply, 1992:23-27.

[11] Bajpai P, Singh SN. Strategic bidding in network constrained electricity markets using FAPSO. International Journal of Energy Sector Management, 2008; 2(2):274-296.

[12] Kazarlis SA, Bakirtzis AG, Petridis V. A genetic algorithm solution to the unit commitment problem. IEEE transaction on Power Systems,1996; 11(1):83-90. 